\title{
ON THE REALIZABILITY OF SINGULAR COHOMOLOGY GROUPS'
}

\section{DANIEL M. KAN AND GEORGE W. WHITEHEAD}

Let $H_{n}(X)$ and $H^{n}(X)$ be the integral singular homology and cohomology groups of a space $X$ and let $\xi$ be the category of abelian groups. Then it is well known that for every sequence $\left(A_{1}, A_{2}, \cdots, A_{n}, \cdots\right)$ with the $A_{n} \in \mathcal{G}$, there exists a space $X$ such that $H_{n}(X) \approx A_{n}$ for all $n>0$. We will show that the analogous statement for cohomology is false. In fact we prove:

Theorem. There exists no space $X$ and integer $n \geqq 1$ such that $H^{n-1}(X)=0$ and $H^{n}(X) \approx Z_{0}$ (the additive group of the rationals).

In the proof the following results will be used.

(a) $Z_{0}$ has no nontrivial direct sum decomposition (trivial).

(b) $\operatorname{Hom}(A, Z)$ is not divisible for any $A \in \mathcal{G}$ (trivial).

(c) ${ }^{2}$ Let $A \in \mathrm{g}$ and $\operatorname{Hom}(A, Z)=0$. Then $\operatorname{Ext}(A, Z)$ is divisible if and only if $A$ is torsionfree and $\operatorname{Ext}(A, Z)$ is torsionfree if and only if $A$ is divisible.

Proof. We will write $\operatorname{Hom} B$ and $\operatorname{Ext} B$ instead of $\operatorname{Hom}(B, Z)$ and $\operatorname{Ext}(B, Z)$. For any integer $m>1$ consider the exact sequence

$$
0 \rightarrow{ }_{m} A \rightarrow A \stackrel{m}{\rightarrow} A \rightarrow A_{m} \rightarrow 0 .
$$

Because Hom $A=\operatorname{Hom}_{m} A=0$ application of the functor Ext yields the exact sequence

$$
0 \rightarrow \operatorname{Ext} A_{m} \rightarrow \operatorname{Ext} A \stackrel{m}{\rightarrow} \operatorname{Ext} A \rightarrow \operatorname{Ext}_{m} A \rightarrow 0
$$

and hence Ext $A_{m}={ }_{m}(\operatorname{Ext} A)$ and $\operatorname{Ext}{ }_{m} A=(\operatorname{Ext} A)_{m}$. For any torsion group $T$, Ext $T=0$ if and only if $T=0$. Hence ${ }_{m} A=0$ if and only if Ext ${ }_{m} A=(\text { Ext } A)_{m}=0$ and $A_{m}=0$ if and only if Ext $A_{m}={ }_{m}($ Ext $A)$ $=0$. The proposition now follows from the fact that a group $B \in \mathcal{G}$ is torsionfree if and only if $m B=0$ for all $m>1$ and that $B$ is divisible if and only if $B_{m}=0$ for all $m>1$.

(d) If $A \in \mathcal{G}$ is torsionfree and divisible, then $A$ is a vector space over $Z_{0}$ (trivial).

Received by the editors March 25, 1960.

1 This research was partially supported by the Office of Ordnance Research, U. S. Army.

2 The first half of this proposition was proved by R. J. Nunke (Illinois J. Math. vol. 3 (1959) p. 230) without the restriction $\operatorname{Hom}(A, Z)=0$. 
(e) If $j: A \rightarrow B \in \mathcal{G}$ is a monomorphism, then $\operatorname{Ext}(j, Z): \operatorname{Ext}(B, Z)$ $\rightarrow \operatorname{Ext}(A, Z)$ is an epimorphism (trivial).

(f) $\operatorname{Ext}\left(Z_{0}, Z\right)$ is not countable.

PROof. The exact sequence $0 \rightarrow Z \rightarrow Z_{0} \rightarrow Z_{0} / Z \rightarrow 0$ induces an exact sequence

$$
0 \rightarrow \operatorname{Hom}\left(Z_{0}, Z_{0}\right) \rightarrow \operatorname{Hom}\left(Z_{0}, Z_{0} / Z\right) \rightarrow \operatorname{Ext}\left(Z_{0}, Z\right) \rightarrow 0 .
$$

$\operatorname{As} \operatorname{Hom}\left(Z_{0}, Z_{0}\right) \approx Z_{0}$ is countable it suffices to show that $\operatorname{Hom}\left(Z_{0}, Z_{0} / Z\right)$ is not. For every sequence $a_{1}, a_{2}, \cdots, a_{n}, \cdots \in Z_{0} / Z$ such that $n a_{n}=a_{n-1}$ for all $n$ there clearly is a homomorphism $f: Z_{0} \rightarrow Z_{0} / Z$ such that $f(1 / n !)=a_{n}$. As the set of these sequences is not countable neither is $\operatorname{Hom}\left(Z_{0}, Z_{0} / Z\right)$.

Proof of THe Theorem. Let $X$ be a space such that $H^{n-1}(X)=0$ and $H^{n}(X) \approx Z_{0}$. By the universal coefficient theorem

$$
\begin{aligned}
0 & =H^{n-1}(X) \approx \operatorname{Hom}\left(H_{n-1}(X), Z\right)+\operatorname{Ext}\left(H_{n-2}(X), Z\right) \\
Z_{0} & \approx H^{n}(X) \approx \operatorname{Hom}\left(H_{n}(X), Z\right)+\operatorname{Ext}\left(H_{n-1}(X), Z\right) .
\end{aligned}
$$

Hence $\operatorname{Hom}\left(H_{n-1}(X), Z\right)=0$, by (a) and (b) $Z_{0} \approx \operatorname{Ext}\left(H_{n-1}(X), Z\right)$ and thus, by (c) $H_{n-1}(X)$ is torsionfree and divisible. But then (d), (e) and (f) imply that $\operatorname{Ext}\left(H_{n-1}(X), Z\right)$ is not countable which is a contradiction, q.e.d.

REMARK. It is not known whether in the theorem the hypothesis $H^{n-1}(X)=0$ can be omitted, i.e., whether $Z_{0}$ can be a singular cohomology group at all.

Massachusetts Institute of Technology 